\title{
An analysis of lubricating medium flow through unsymmetrical lubricating gap of conical slide bearing
}

\author{
Mariusz Koprowski, \\ Gdynia Maritime University
}

\begin{abstract}
This paper presents a computer analysis of lubricating medium flow through unsymmetrical lubricating gap of conical slide bearing. Numerical calculations were carried out with the use of the software Matlab 7.1 and Mathematica 5.2 for example conical slide bearings of different values of cone apex angles of pin and sleeve and set values of relative eccentricity and skewing angle as well as dimensionless bearing length equal to 1.
\end{abstract}

Keywords : unsymmetrical lubricating gap, conical slide bearing, numerical calculations, hydrodynamic pressure distribution, load carrying capacity

\section{INTRODUCTION}

Conical bearings were considered as sliding friction units by the following authors: $[1,3,4,6,7,11,13,15]$. In the literature sources $[4,6,11,13,15]$ a simplified one-dimensional theoretical flow model of oil as lubricating medium in gap of conical slide bearings was considered. The papers [1] and [7] dealt with gas lubrication of conical slide bearings. In all the above mentioned papers their authors assumed axially symmetrical flow of lubricating medium. Such assumption is a rough simplification of real lubrication conditions as it does not take into account two crucial facts. Firstly, cones of the pin and sleeve forming the conical bearing can have different values of apex angle. Secondly, a skewing of the pin within the sleeve may occur as a result of their non-parallel axes. The skewing results from inaccuracy of machining and assembling operations or deformation of sleeve and pin due to mechanical and thermal loading. In the opinion of this author the facts should not be neglected in analysing the lubrication process of conical slide bearings as they significantly impact the lubricating medium flow through the gap of slide bearing, which changes this way its operational parameters.

In the presented lubricating gap model a novelty is the taking into consideration of the above mentioned aspects. Moreover, the presented mathematical models concerning lubrication analysis of conical slide bearings make it possible to directly pass from the elaborated model of conical slide bearings to the known models of cylindrical journal slide bearings.

\section{GEOMETRICAL AND MATHEMATICAL MODEL OF LUBRICATING GAP}

Fig. 1 presents geometry of the conical slide bearing considered in this paper, where the pin and sleeve axes are inclined to each other by the angle $v$ and the pin and sleeve centres are shifted by the bearing eccentricity e. The angles $\gamma$ and $\gamma_{1}$ are the inclination angles of generatrices of pin and sleeve cones.
In order to prevent contact of metal surfaces of pin and sleeve and resulting seizure of the bearing the following condition is to be satisfied : $\gamma-\gamma_{1} \geq 0$

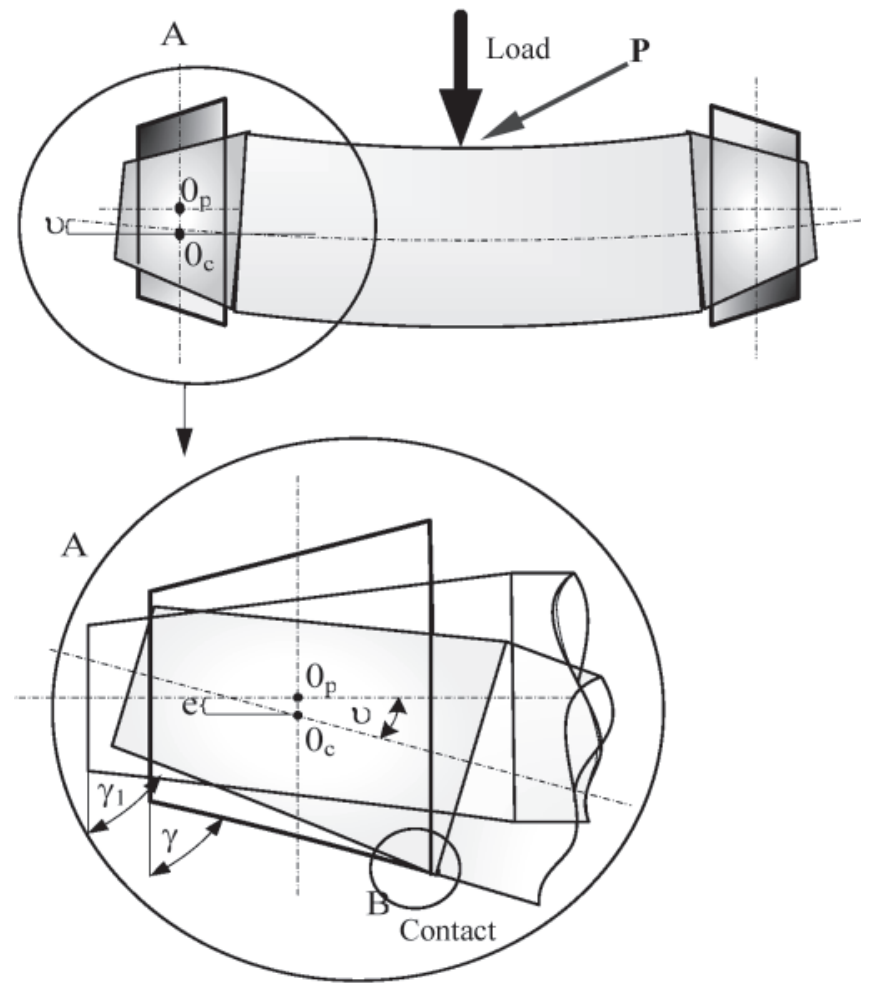

Fig. 1. Geometrical model of conical slide bearing, $v\left[0^{\circ}\right]$ - skewing angle, $\boldsymbol{O}_{p}, \boldsymbol{O}_{c}-$ centres of sleeve and pin, respectively, $e[\mathrm{~m}]$ - bearing eccentricity

The skewing shown in Fig. 1 results from the applied load P. Fig. 1 simultaneously serves as a graphical illustration of the considered model of conical slide bearing. In the model the axis 
skewing angle occurs in the plane consisting the centre line of pin and sleeve. The point B (Fig. 1) is the theoretical contact point of pin and sleeve. Simultaneously, its position determines the maximum value of skewing angle of a given bearing.

The dimensional equation which describes the lubricating gap in the conical slide bearing and takes into consideration the skewing of the sleeve as well as that the pin and sleeve cones forming the bearing can have different values of cone apex angles, is expressed as follows :

$$
\begin{gathered}
\mathrm{h}\left(\varphi, \mathrm{x}, \gamma, \gamma_{1}, \mathrm{v}\right)=\mathrm{c}(1+\lambda \cos \varphi) \Gamma+ \\
+\mathrm{x}(\operatorname{tg} v)(\cos \varphi)+(\mathrm{x}+\mathrm{L}) \sin \left(\gamma-\gamma_{1}\right)
\end{gathered}
$$

where :

$\mathrm{c}[\mathrm{m}] \quad-$ radial clearance

$\Gamma=1 / \sin (\gamma), 0 \leq \gamma \leq \pi$

$\lambda=\mathrm{e} / \mathrm{c}[-]-$ relative eccentricity

$\varphi, \mathrm{x} \quad-$ conical coordinates

$\mathrm{L}[\mathrm{m}] \quad-$ half-length of the pin cone generatrix.

To generalize the equation (1) which describes gap height change the relationships connecting together dimensionless and dimensional quantities are assumed as follows:

$$
\mathrm{h}_{1}=\frac{\mathrm{h}}{\mathrm{c}} ; \mathrm{L}_{1}=\frac{\mathrm{L}}{\mathrm{R}_{0}} ; \mathrm{x}=\mathrm{Lx}_{1}=\mathrm{L}_{1} \mathrm{R}_{0} \mathrm{x}_{1}
$$

where:

$\mathrm{L}_{1}[-] \quad$ - dimensionless length of bearing,

$\mathrm{x}_{1} \quad$ - dimensionless conical coordinate,

$\mathrm{R}_{0}[\mathrm{~m}]$ - radius measured along the coordinate $\mathrm{x}$ at the halfheight of pin cone.

On insertion of the relations (2) into the equation (1) which describes the gap-height change the following dimensionless form of the gap height is obtained:

$$
\begin{gathered}
\mathrm{h}\left(\varphi, \mathrm{x}_{1}, \gamma, \gamma_{1}, \nu\right)=\underbrace{(1+\lambda \cos \varphi) \Gamma}_{1}+ \\
+\underbrace{\Psi \mathrm{L}_{1} \mathrm{x}_{1} \cos \varphi}_{2}+\underbrace{\mathrm{YL}_{1} \mathrm{x}_{1}}_{3}
\end{gathered}
$$

where:

$$
\begin{gathered}
\Psi \equiv(\operatorname{tg} v) / \psi, 0 \leq \Psi \leq 1, \gamma \equiv\left[\sin \left(\gamma-\gamma_{1}\right)\right] / \psi, 0 \leq \gamma \leq 1, \\
\Gamma=1 / \sin \gamma, \gamma \neq 0, \psi=\mathrm{c}_{0} \mathrm{R}_{0}, \psi \approx 0.001
\end{gathered}
$$

The gap model (3) can be compared with that of the slide bearing whose pin and sleeve surfaces are assumed perfectly smooth and stiff. Also, deformations of bearing surfaces, which result from oil pressure and temperature, are not taken into consideration.

The term 1 of the gap-height mathematical model (3) for $\Gamma=1$ describes the lubricating gap relevant for typical journal slide bearings in the case when the inclination angles of pin and sleeve generatrices against the vertical are equal to $90^{\circ}$

If value of the angle is less than $90^{\circ}$ the term describes the gap of the journal bearing whose pin is skewed. The term 2 determines influence of the skewing angle on the lubricating gap height in the conical slide bearing (Fig. 3a). If the angle $v=0$ then $\Psi=0$ and in consequence the term equals zero. The term 3 of the lubricating gap equation (3) describes solely influence of the cone apex angles of pin and sleeve on the lubricating gap height in conical slide bearing. If the angles $\gamma$ and $\gamma_{1}$ are equal to each other then the term equals zero and the lubricating gap height is constant along the coordinate $\mathrm{x}_{1}$.

Fig. 2 and 3 graphically illustrate changes of lubricating gap height of conical slide bearing, depending on the relative eccentricity $\lambda$, the pin skewing angle $v$ against the sleeve axis, and different values of the inclination angles $\gamma$ and $\gamma_{1}$ against the vertical, of generatrices of pin and sleeve cones.
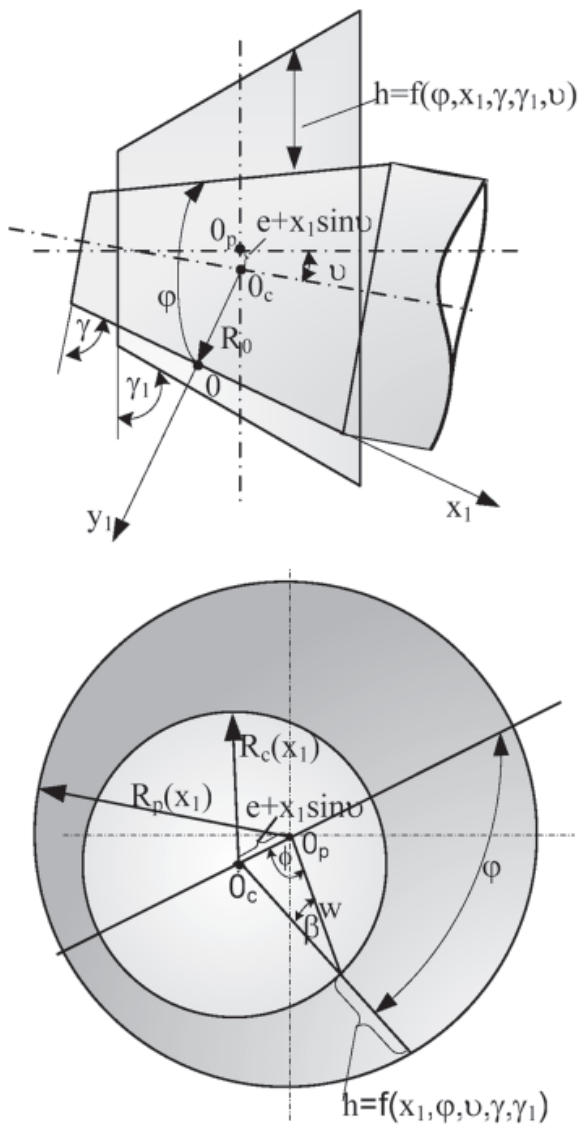

Fig. 2. Geometrical model of conical slide bearing in the conical coordinate frame: a) Longitudinal cross-section, b) Transverse cross-section; $\boldsymbol{0}$ - the centre of the coordinate frame; $\boldsymbol{R}_{0}$ - pin radius measured in the point $0 ; \boldsymbol{\varphi}, \boldsymbol{\beta}$-auxiliary angles $; \boldsymbol{R}_{p}\left(\boldsymbol{x}_{\boldsymbol{p}}\right), \boldsymbol{R}_{c}\left(\boldsymbol{x}_{\boldsymbol{p}}\right)$-sleeve and pin radiuses in function of the coordinate $\boldsymbol{x}_{1}$.

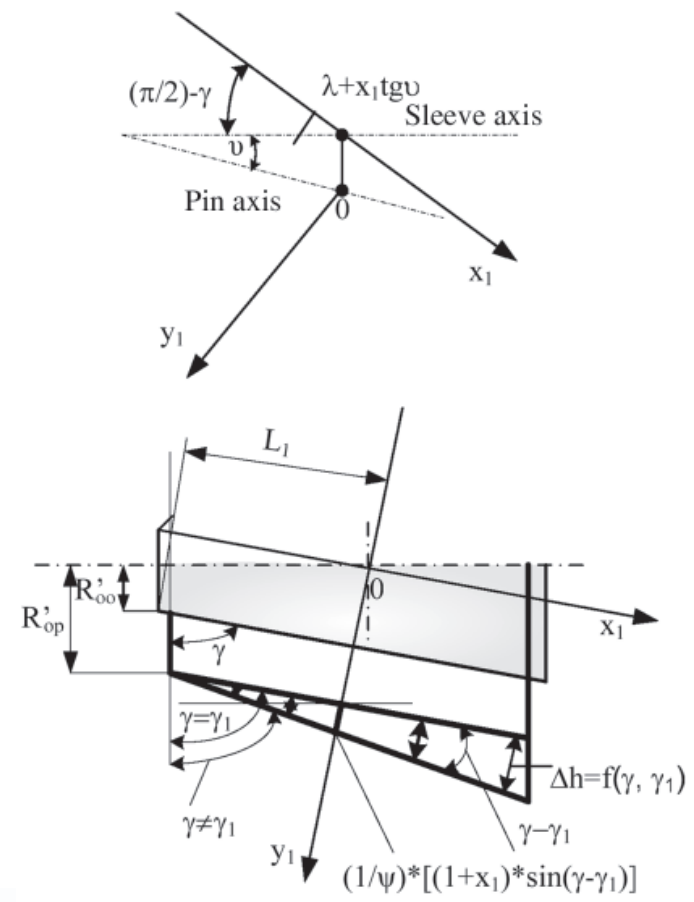

Fig. 3. Details of the angles appearing in the conical slide bearing a) influence of the skewing angle on the lubricating gap; b) influence of the apex angles of generatrices of pin and sleeve cones on the lubricating gap;

$\Delta \boldsymbol{h}$-lubricating gap increase due to difference of the pin and sleeve apex angles ; $\boldsymbol{R}^{,}, \boldsymbol{R}^{\prime}$ - pin and sleeve radiuses measured in the cut-off places of the cones. 


\section{ANALYSIS OF SKEWING IN THE LUBRICATING GAP MODEL}

Allowable values of the skewing angle $v$ (Fig. 2) of the pin and sleeve axes in conical slide bearing depend on values of the relative eccentricity $\lambda$ and the pin and sleeve cone apex angles. The limit value of the skewing angle $v$ at which metal-to-metal contact of the pin and sleeve occurs in the bearing (Fig. 1), can be determined by using the following expression :

$$
v=\operatorname{arctg}\left\{\frac{\psi}{L_{1}} \Gamma[(1-\lambda)+2 Y \sin \gamma]\right\}
$$

where:

$$
\Gamma=1 / \sin \gamma, \gamma \neq 0, \Psi \equiv\left[\sin \left(\gamma-\gamma_{1}\right)\right] / \psi
$$

To check correctness of the mathematical model of lubricating gap , (3), as well as the expression (4) a computer analysis was performed by means of the software Mathematica 5.0. The simulation was carried out for several selected values of the eccentricity $\lambda$, angles $\gamma, \gamma_{1}$ and skewing angle $v$. The calculated results of gap changes, obtained this way are graphically presented in Fig. 4. The limit values of the skewing angle $v$ for the assumed values of the parameters $\lambda, \gamma$ and $\gamma_{1}$ are presented in Tab. 1 .

Tab. 1. Values of the pin skewing angle in the conical slide bearing, determined from the formula (4) for the assumed values of the slide bearing parameters

\begin{tabular}{|c|c|c|c|c|c|}
\hline No. & $\lambda[-]$ & $\gamma\left[^{\circ}\right]$ & $\gamma_{1}\left[^{0}\right]$ & $\gamma-\gamma_{1}\left[^{0}\right]$ & $v\left[^{0}\right]$ \\
\hline 1. & 0.2 & 90 & 90 & 0 & 0.0458366 \\
\hline 2. & 0.2 & 88.2 & 88.2 & 0 & 0.0458584 \\
\hline 3. & 0.4 & 87.37 & 86.5 & 0 & 0.0344135 \\
\hline 4. & 0.4 & 87.37 & 86.49 & 0.01 & 0.0544135 \\
\hline $\mathbf{5 .}$ & 0.6 & 86.53 & 86.53 & 0 & 0.0229602 \\
\hline $\mathbf{6 .}$ & 0.6 & 86.53 & 86.529 & 0.01 & 0.0429602 \\
\hline 7. & 0.8 & 85.71 & 85.71 & 0 & 0.0114913 \\
\hline $\mathbf{8 .}$ & 0.8 & 85.71 & 85.70 & 0.01 & 0.0314913 \\
\hline
\end{tabular}

\section{LOAD CARRYING CAPACITY OF SLIDE BEARING}

Apart from friction force and friction coefficient, load carrying capacity is the main parameter which characterizes operational features of slide bearings. To determine slide bearing's capacity it is necessary to analyze equilibrium conditions of normal and tangential forces acting within oil film (Fig. 5).

According to the notation used in Fig. 5 and the known definitions of bearing capacity forces [2], [5] the following expressions for the capacity force components can be given:

The dimensionless transverse component of capacity force, $\mathrm{C}_{1 \mathrm{p}}$ :

$$
\mathrm{C}_{1 \mathrm{p}}=\sqrt{\mathrm{C}_{1 \mathrm{pX}}^{2}+\mathrm{C}_{1 \mathrm{pY}}^{2}}
$$

where:

$$
\begin{aligned}
& \mathrm{C}_{1 \mathrm{pX}}=\int_{-1}^{+1} \int_{0}^{\varphi_{\mathrm{k}}} \mathrm{p}_{1} \cos \varphi \sin (\gamma-v) \mathrm{d} \varphi \mathrm{d} \mathrm{x}_{1} \\
& \mathrm{C}_{1 \mathrm{pY}}=\int_{-1}^{+1 \varphi_{\mathrm{k}}} \int_{0} \mathrm{p}_{1} \sin \varphi \sin (\gamma-v) \mathrm{d} \varphi \mathrm{d} \mathrm{x}_{1}
\end{aligned}
$$

a)
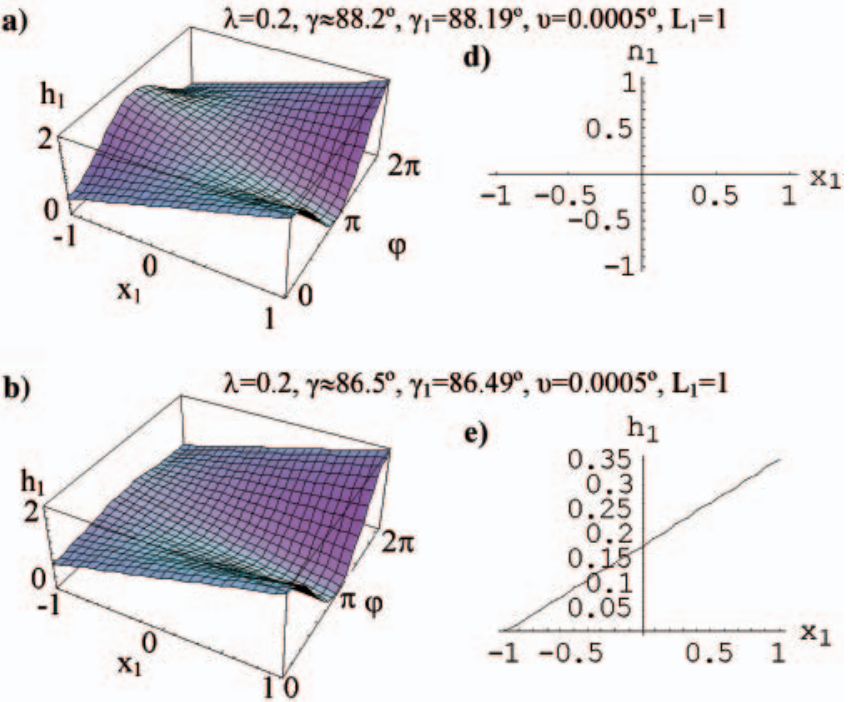

e)
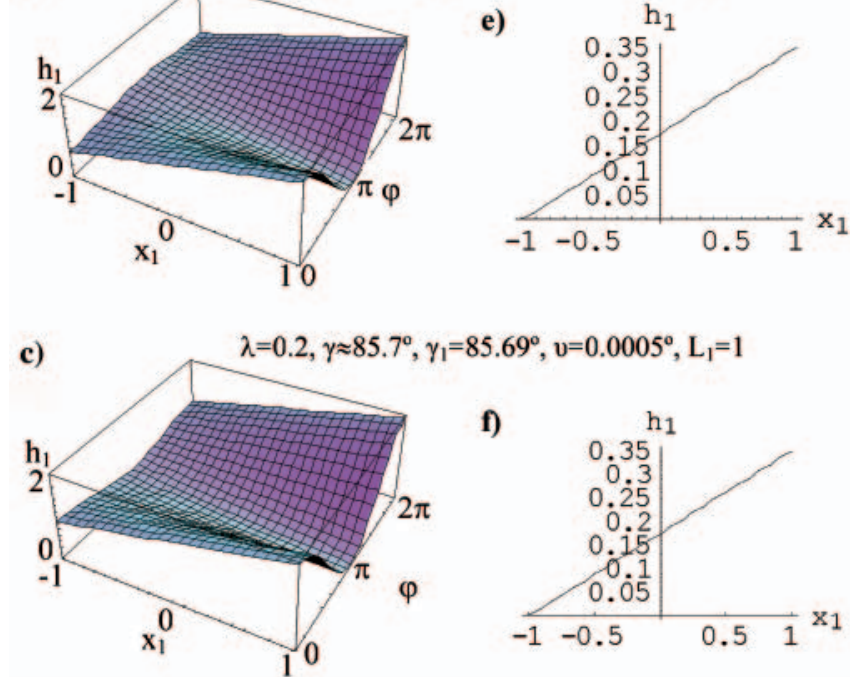

Fig. 4. Lubricating gap changes in conical slide bearing for several values of the relative eccentricity $\lambda ; \boldsymbol{a}), \boldsymbol{b}), \boldsymbol{c})$-developed view of the gap height. $\boldsymbol{d}), \boldsymbol{e}), \boldsymbol{f})$ - increase of the lubricating gap height due to difference of the angles $\gamma$ and $\gamma_{l}$.
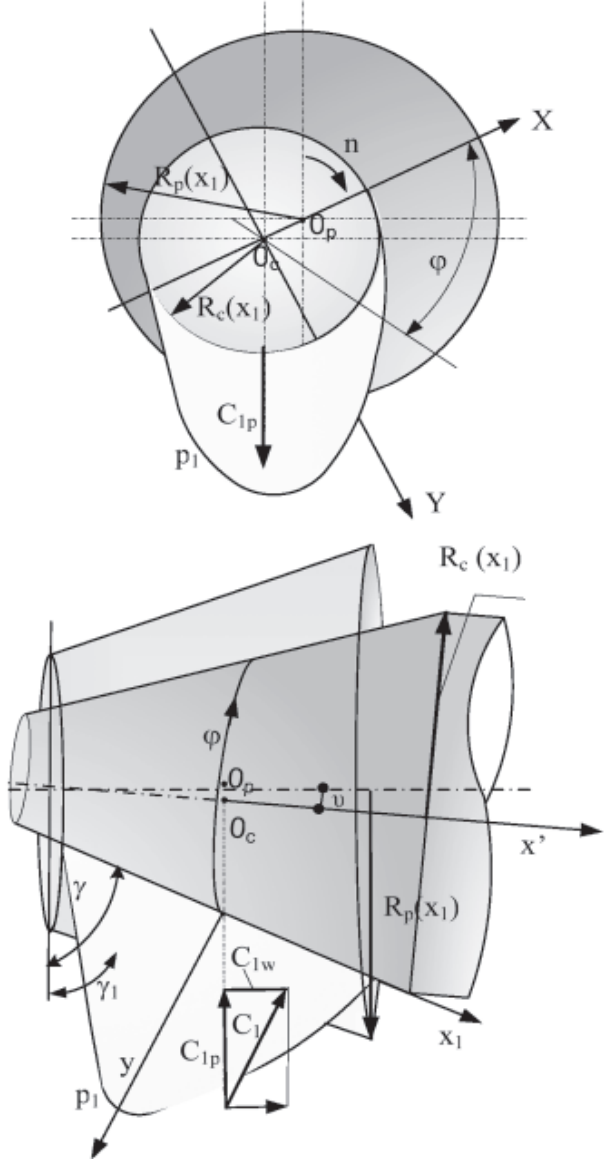

Fig. 5. Schematic diagram of position of resultant capacity force in conical slide bearing ; $\boldsymbol{C}_{1 p}$ - dimensionless transverse component of capacity force ; $\boldsymbol{C}_{\boldsymbol{I}_{\boldsymbol{w}}}$ - dimensionless longitudinal component of capacity force. 
> The dimensionless longitudinal component of capacity force, $\mathrm{C}_{1 \mathrm{w}}$ :

$$
\mathrm{C}_{1 \mathrm{w}}=\sqrt{\mathrm{C}_{1 \mathrm{wX}}^{2}+\mathrm{C}_{1 \mathrm{w} Y}^{2}}
$$

where:

$$
\begin{aligned}
& C_{1 \mathrm{wX}}=\int_{-1}^{+1 \varphi_{\mathrm{k}}} \mathrm{p}_{0} \cos \varphi \sin (\gamma-v) \mathrm{d} \varphi \mathrm{d} \mathrm{x}_{1} \\
& \mathrm{C}_{1 \mathrm{w} \mathrm{Y}}=\int_{-1}^{+1 \varphi_{\mathrm{k}}} \int_{0} \mathrm{p}_{1} \sin \varphi \sin (\gamma-v) \mathrm{d} \varphi \mathrm{d} \mathrm{x}_{1}
\end{aligned}
$$

The total (resultant) capacity force of conical slide bearing, $\mathrm{C}_{1}$, is the geometrical sum of the transverse force $\mathrm{C}_{1 \mathrm{p}}$ and longitudinal force $\mathrm{C}_{1 \mathrm{w}}$ :

$$
\mathrm{C}_{1}=\sqrt{\mathrm{C}_{1 \mathrm{p}}^{2}+\mathrm{C}_{1 \mathrm{w}}^{2}}
$$

And, values of the forces $\mathrm{C}_{1 \mathrm{p}} \mathrm{iC}_{1 \mathrm{w}}$ depend on the pin cone apex angle, skewing angle and hydrodynamic pressure.

To determine the total force $\mathrm{C}_{1}(11)$ of a given bearing it is necessary to know value of the dimensionless hydrodynamic pressure $\mathrm{p}_{1}$ which can be determined from the following formula [9]:

$\frac{1}{\mathrm{U}_{1}^{2}} \frac{\partial}{\partial \varphi}\left(\frac{\mathrm{h}_{1}^{3}}{\eta_{1}} \frac{\partial \mathrm{p}_{1}}{\partial \varphi}\right)+\frac{1}{\mathrm{~L}_{1}^{2}} \frac{\partial}{\partial \mathrm{x}_{1}}\left(\frac{\mathrm{h}_{1}^{3}}{\eta_{1}} \frac{\partial \mathrm{p}_{1}}{\partial \mathrm{x}_{1}}\right)=6 \mathrm{U}_{1} \frac{\partial \mathrm{h}_{1}}{\partial \varphi}$

where:

$$
\mathrm{U} 1=\mathrm{L} 1(1+\mathrm{x} 1) \cos \gamma
$$

The differential equation (12) obtained by imposing relevant boundary conditions on the transverse component of velocity vector (measured perpendicularly to pin's conical surface) in the set of fundamental equations describes lubricating medium flow through the conical slide bearing gap [9].

Values of the distribution of the hydrodynamic pressure $\mathrm{p}_{1}$ and the capacity force $\mathrm{C}_{1}$ were numerically calculated by means of the software MATLAB $7.1[8,10]$. The calculations were based on the finite differences method and the author's calculation procedures. The example calculation results of the hydrodynamic pressure distribution for the relative eccentricity $\lambda=0.2$ are presented in Fig. 6, where Fig. 6a) shows hydrodynamic pressure changes within the journal slide bearing gap, and Figs. 6 b,c present the pressure distribution within the journal slide bearing gap. Fig. 7 presents changes of the capacity force $C_{1}$ in function of the relative eccentricity $\lambda$ for several selected values of the cone apex angles of pin and sleeve at a constant value of the skewing angle $v$.

\section{FINAL REMARKS AND CONCLUSIONS}

From Fig. 1 and the numerical calculation results it can be observed that the pin skewing in the conical slide bearing sleeve leads to concentration of pressure, especially on its edge. The greater values of the skewing angle $v$, the relative eccentricity $\lambda$ and the difference of the cone apex angles $\gamma$ and $\gamma_{1}$ (for conical slide bearings), the greater pressure in question.

The presented mathematical model of lubricating gap (3) is general as it determines the lubricating gap of both the conical slide bearings and journal slide bearings (when the angles $\gamma$ and $\gamma_{1}$ are assumed equal 90 ${ }^{\circ}$ (Fig. 6 and 7).
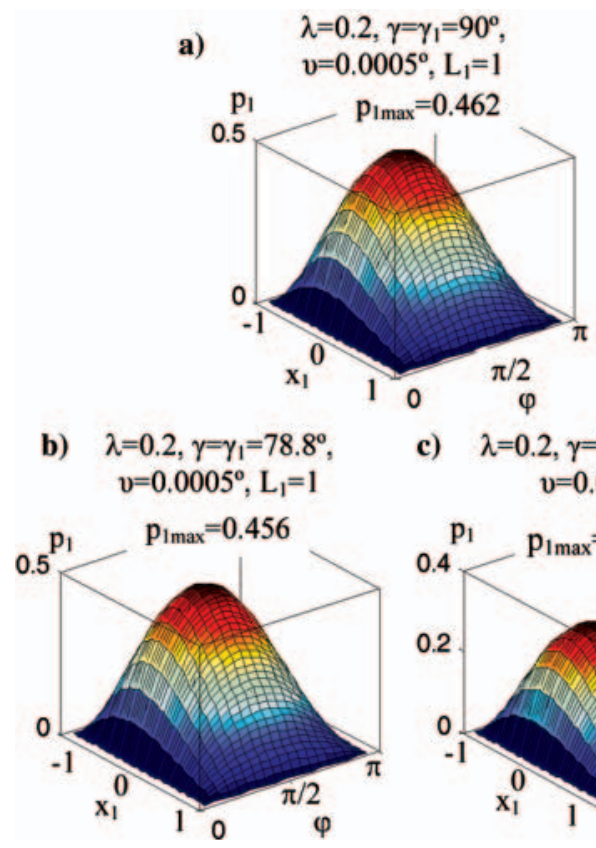

c) $\lambda=0.2, \gamma=78.8^{\circ}, \gamma_{1}=78.79^{\circ}$,

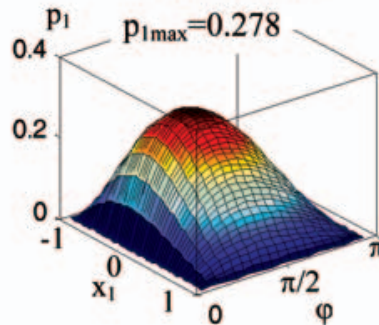

Fig. 6. Pressure distribution in slide bearing. a) journal one; b), c)-conical one.
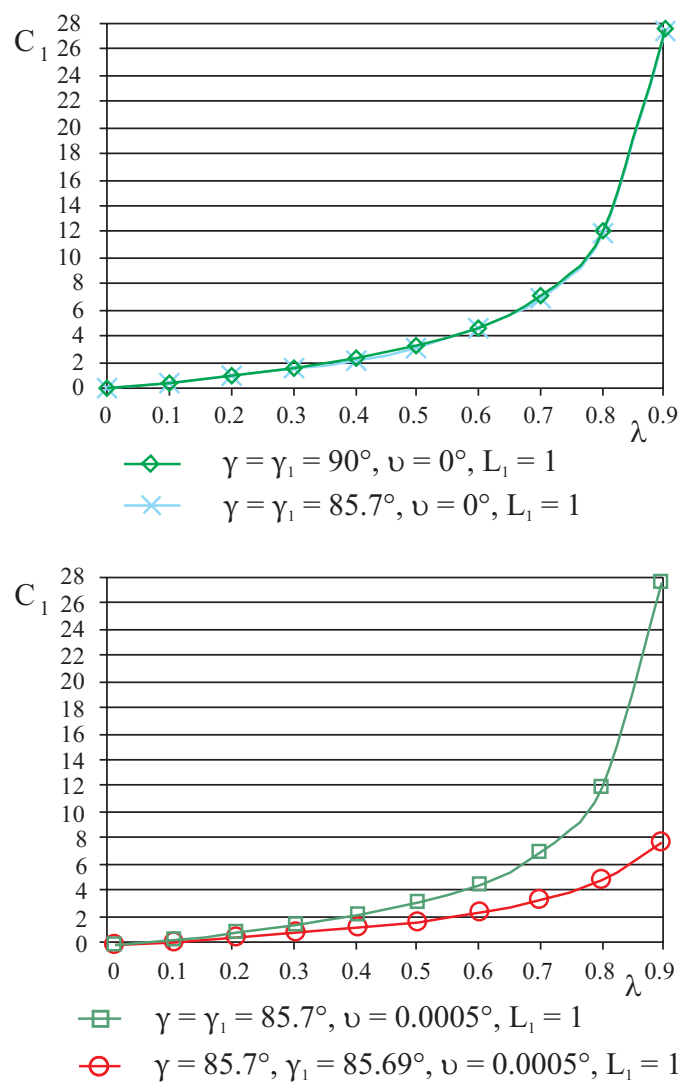

Fig. 7. Diagram of changing values of the total capacity force $C_{I}$ in slide bearing

The data presented in Tab. 1 show that the conical slide bearings having an unsymmetrical lubricating gap $\left(\gamma-\gamma_{1}\right)$ make greater limit values of the skewing angles $v$ acceptable as compared with those for conical slide bearings having symmetrical gap constant along the gearing $\left(\gamma-\gamma_{1}\right)$. The phenomenon results from an additional increase of lubricating gap height by the value $\Delta \mathrm{h}$ (Fig. 3) resulting from difference of the angles $\gamma$ and $\gamma_{1}$.

Fig. 6 shows that the hydrodynamic pressure distribution within journal slide bearings s well as conical ones having the gap symmetrical and constant along the bearing take 
similar values at the same relative eccentricity $\lambda$. Increasing difference of the cone apex angles of pin and sleeve results in decreasing the pressure $p_{1}$ within conical slide bearings (Fig. $6 \mathrm{c}$ ). The phenomenon is associated with a suction action of conical pin rotating within the bearing exerted onto lubricating medium flowing through the gap.

From the diagram of lifting forces (Fig. 7) it results that the load carrying capacity of conical slide bearings significantly decreases along with increasing the difference of the cone apex angles of pin and sleeve.

\section{NOMENCLATURE}

$\mathrm{c}=\mathrm{R}_{\mathrm{p}}-\mathrm{R}_{\mathrm{c}}-$ radial clearance $[\mathrm{m}]$

$\mathrm{C}_{1} \quad$ - dimensionless value of slide bearing capacity force [-]

$\mathrm{C}_{1 \mathrm{p}} \quad-$ dimensionless value of transverse component of slide bearing capacity force [-]

$\mathrm{C}_{1 \mathrm{pX}} \quad-$ dimensionless value of transverse component of slide bearing capacity force along $\mathrm{X}$ - direction [-]

$\mathrm{C}_{1 \mathrm{pY}} \quad$ dimensionless value of transverse component of slide bearing capacity force along Y-direction [-]

$\mathrm{C}_{1 \mathrm{w}} \quad-$ dimensionless value of longitudinal component of slide bearing capacity force [-]

$\mathrm{C}_{1 \mathrm{wX}} \quad-$ dimensionless value of longitudinal component of slide bearing capacity force along $\mathrm{X}$ - direction [-]

$\mathrm{C}_{1 \mathrm{wY}} \quad-$ dimensionless value of longitudinal component of slide bearing capacity force along Y - direction [-]

$\mathrm{e}=\mathrm{O}_{\mathrm{p}}-\mathrm{O}_{\mathrm{c}}-$ eccentricity $[\mathrm{m}]$

$\mathrm{h} \quad \mathrm{p} \quad-$ dimensional value of lubricating gap height [m]

$\mathrm{h}_{1} \quad-$ dimensionless value of lubricating gap height [-]

$\mathrm{L} \quad-$ dimensional value of slide bearing length [m]

$\mathrm{L}_{1}=\mathrm{L} / \mathrm{R}_{\mathrm{o}}-$ dimensionless value of slide bearing length [-]

$\mathrm{O}_{\mathrm{c}} \quad-$ pin centre

$\mathrm{O}_{\mathrm{p}}^{\mathrm{c}} \quad-$ sleeve centre

$\mathrm{p}_{1}{ }^{\mathrm{p}} \quad-$ dimensionless value of hydrodynamic pressure in bearing gap [-]

$\mathrm{p}_{\mathrm{o}} \quad-$ characteristic value of hydrodynamic pressure [ $\left.\mathrm{MPa}\right]$

$\mathrm{R}_{\mathrm{c}} \quad-$ radius of slide bearing pin [m]

$\mathrm{R}^{\prime}{ }_{\mathrm{oc}} \quad-$ pin radius measured at the cut of slide bearing cone $[\mathrm{m}]$

$\mathrm{R}^{{ }^{\circ}{ }_{\text {op }}} \quad-$ sleeve radius measured at the cut of slide bearing sleeve cone $[\mathrm{m}]$

$\mathrm{R}_{0} \quad-$ pin radius measured at the height of the assumed centre of coordinate frame $[\mathrm{m}]$

$\mathrm{R} \quad-$ radius of slide bearing sleeve $[\mathrm{m}]$

$\mathrm{U}_{1}^{\mathrm{p}}=\mathrm{L}_{1}\left(1+\mathrm{x}_{1}\right) \cos \gamma-$ dimensional value of circumferential velocity of pin [-]

$\mathrm{X}, \mathrm{Y}-$ coordinates of auxiliary coordinate frame

$\mathrm{Y} \equiv\left[\sin \left(\gamma-\gamma_{1}\right)\right] / \psi$

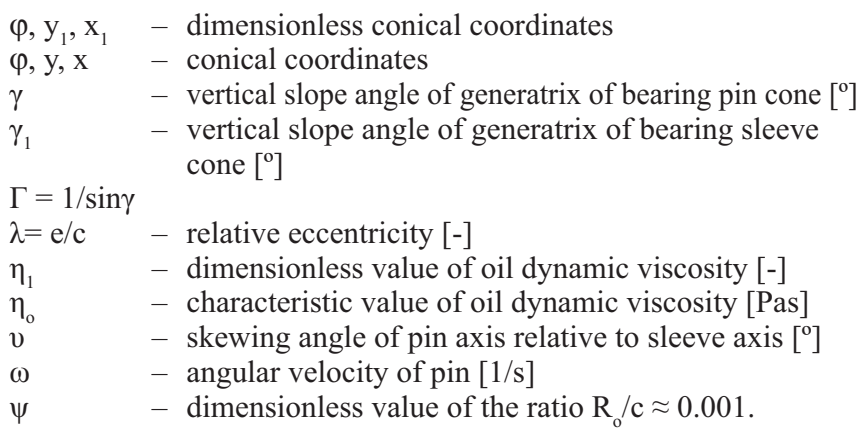

\section{BIBLIOGRAPHY}

1. Apanasewicz S., Kazimierski Z., Lewandowski J, Szaniewski A.: The flow of the gas layer between two conical surfaces. Fluid Dynamics Transactions Vol. 5, Part II, PWN, Warszawa 1971

2. Burcan J.: Bearing of hyperboloidal sleeve (in Polish). ZEM no. 3-4, 1973

3. Fuller D.D.: Theory and practice of lubrication (in Polish). PWT, Warszawa 1960

4. Gottwald I.,Vieveg R.: Berechnung und Modellversuche an Wasser-und Luftlagern. Zeit. Angewandte Physik Bd. II, H. 11.1950,

5. Hebda M., Wachal A.: Tribology (in Polish). WNT, Warszawa 1980

6. Janiszewski R.: Theoretical analysis of magnetohydrodynamic flow of lubricating medium through conical slide bearing (in Polish). Doctoral thesis, Lublin University of Technology -Mining \& Metallurgy Academy (AGH), Kraków 1980

7. Kazimierski Z.: Unsteady flow in thin layer of viscous gas between two non-coaxial cones (in Polish). Publ. of Lodz University of Technology, Heat Machines 75, 1972

8. Kazimierski Z.: Essentials of fluid mechanics and methods of computer simulation of flow (in Polish). Publ. of Lodz University of Technology, Łódź 2004

9. Koprowski M.: Hydrodynamic models of lubrication of conical slide bearings in magnetic field (in Polish). Trybologia no. 3, 2006 ,

10.Majchrzak E., Mochnacki B.: Numerical methods. Essentials of theory, practical aspects and algorithms (in Polish). Publ. of Wrocław University of Technology, 1996

11. Szaniewski A.: Flow of viscous incompressible liquid through conical slide bearing gap (in Polish). Publ. of IPP PAN, 15, Warszawa 1970

12. Walicki E.: Load carrying capacity of conical slide bearing (in Polish). Scientific Bulletins (Zeszyty Naukowe) no.7, Mechanics and Structures (Mechanika- konstrukcje) 4, Bydgoszcz High School of Engineering (WSI), 1973

13. Wierzcholski K .: Flow of non - Newtonian power law lubricant through the conical bearing gap. Acta Mechanica 50, 1984 ,

14. Wierzcholski K.: Theory of unconventional lubrication of slide bearings (in Polish). Publ. of Szczecin University of Technology (Wydawnictwo Uczelniane Politechniki Szczecińskiej), Szczecin, 1995

15.Wierzcholski K., Nowak Z.: Influence of a temperature dependent lubricant consistency on the capacity of a conical journal bearing. Proc. of the Congress on Rheology, Acapulco 1984

\section{CONTACT WITH THE AUTHOR}

Mariusz Koprowski, Ms. C.

Cathedral of Bases of the Technique,

Gdynia Maritime University

Morska 81/87

81-225 Gdynia, POLAND

e-mail : mkoprows@am.gdynia.pl

tel.: (058) 6901-483 